\title{
Kernos
}

Revue internationale et pluridisciplinaire de religion grecque antique

7| 1994

Varia

\section{De Cnossos à Mycènes : emprunts et syncrétismes en Grèce préhistorique}

\section{Robert Laffineur}

\section{(apenEdition \\ Journals}

\section{Édition électronique}

URL : http://journals.openedition.org/kernos/1102

DOI : 10.4000/kernos. 1102

ISSN : 2034-7871

\section{Éditeur}

Centre international d'étude de la religion grecque antique

\section{Édition imprimée}

Date de publication : 1 janvier 1994

ISSN : 0776-3824

\section{Référence électronique}

Robert Laffineur, "De Cnossos à Mycènes : emprunts et syncrétismes en Grèce préhistorique », Kernos [En ligne], 7 | 1994, mis en ligne le 20 avril 2011, consulté le 21 avril 2019. URL : http:// journals.openedition.org/kernos/1102; DOI : 10.4000/kernos.1102 
Kernos, 7 (1994), p. 131-143.

\section{DE CNOSSOS À MYCÈNES : \\ EMPRUNTS ET SYNCRÉTISMES EN GRÈCE PRÉHISTORIQUE}

On admet traditionnellement que la Grèce mycénienne a, en matière de croyances et de pratiques religieuses, comme dans bien d'autres domaines de l'activité matérielle, été dominée par l'influence de la Crète minoenne. L'observation a été longuement argumentée et reste, bien sûr, largement valable aujourd'hui. Mon intention n'est pas de la contredire, mais simplement de la nuancer quelque peu, en mettant mieux en évidence, à côté de ces emprunts, les indices d'un

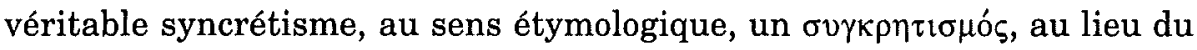
pancrétisme de naguère. Le temps qui m'est imparti ici m'imposera de me limiter à quelques exemples ${ }^{1}$. Je les ai choisis de manière à ce que leur examen contribue à la définition de principes méthodologiques plus généraux.

En l'absence de textes exploitables - sauf, dans une proportion limitée, pour les phases récentes -, la religion mycénienne ne peut être appréhendée, comme la religion crétoise, que par l'identification des vestiges archéologiques d'édifices ou de matériel cultuels ${ }^{2}$ ou par l'interprétation de certaines catégories iconographiques. Cette dernière source doit évidemment être utilisée avec prudence et avec rigueur, le risque de surinterprétation étant important. Mais là n'est pas le propos de notre réunion d'aujourd'hui.

Si l'on s'attache à des scènes dont le contenu cultuel, même s'il ne peut pas toujours être défini avec précision, est incontestable, comme par exemple les scènes qui ornent les longs côtés du célèbre sarcophage peint

1 On ne trouvera donc pas l'équivalent de l'inventaire proposé par P. LÉVÊQUE, Le syncrétisme créto-mycénien, in Les syncrétismes dans les religions de l'antiquité. Colloque de Besançon (22-23 octobre 1973), Leyde, 1975, p. 19-73.

2

Pour les sanctuaires mycéniens, voir récemment K. KILIAN, Mykenische Heiligtümer der Peloponnes, in Kotinos. Festschrift für Erika Simon, Mayence, 1992, p. 10-25. Sur les sources textuelles concernant les sanctuaires, l'étude fondamentale reste celle de St. HILLER, Mykenische Heiligtümer: das Zeugnis der Linear B-Texte, in Sanctuaries and Cults in the Aegean Bronze Age. Proceedings of the First International Symposium at the Swedish Institute in Athens, 12-13 May, 1980, Stockholm, 1981, p. 95-126. 
d'Aghia Triada, on mesure d'emblée une difficulté d'un autre ordre, en rapport direct avec le thème du présent colloque.

Le monument montre d'un côté la présentation d'offrandes au défunt et la préparation du liquide de libation, et de l'autre l'offrande de produits alimentaires et le sacrifice d'un taureau devant un édicule religieux ${ }^{3}$. Ces thèmes ont certes des équivalents et des antécédents en Crète minoenne, dans les scènes d'offrande, de libation et de sacrifice ${ }^{4}$. Le type de monument s'inscrit clairement lui aussi dans une tradition minoenne, celle des sarcophages peints. L'association du sacrifice de taureau au contexte funéraire est enfin attestée en Crète minoenne dans la tombe $\mathrm{A}$ d'Archanes-Phourni, dont la fouille a révélé la présence, dans le passage entre la chambre circulaire principale et la chambre latérale, d'un crâne de taureau ${ }^{5}$. Tout semble donc indiquer qu'on se trouve en présence d'un monument qui illustre des pratiques cultuelles, en l'occurrence des pratiques funéraires, qui sont typiquement minoennes. Et c'est de cette manière que l'on interprète habituellement le sarcophage.

Mais poussons l'enquête plus avant. Le monument d'Aghia Triada n'est pas fabriqué en terre cuite, comme tous les sarcophages minoens, mais en pierre et il est le seul exemplaire connu réalisé dans ce matériau. La décoration peinte y a été exécutée selon une technique qui s'apparente étroitement à celle de la peinture murale et qui semble donc bien confirmer l'appartenance à une tradition technique crétoise. Mais la peinture crétoise est exclusivement immobilière, alors que l'application de la technique dite de la fresque à des documents mobiliers est bien attestée sur le continent mycénien, sur la célèbre tête en stuc peint de Mycènes ${ }^{6}$, la plaque à divinité armée ${ }^{7}$ et la stèle de même provenance ${ }^{8}$, etc. La probabilité d'une identification mycénienne se confirme quand

3 Voir les illustrations dans S. MARINATos et M. HiRmer, Kreta, Thera und das mykenische Hellas, Munich, 1973, pl. XXXI et XXXII, en haut.

4 Pour le sacrifice de taureaux en particulier, voir récemment N. MARINATOs, Minoan Sacrificial Ritual. Cult Practice and Symbolism, Stockholm, 1986.

5 Voir à ce propos I. SAKELLARAKIs, Das Kuppelgrab A von Archanes und das kretisch-mykenische Tieropferritual, in Prähistorische Zeitschrift, 45 (1970), p. 135-219.

6 MaRinatos-HiRmer (supra n. 3), pl. LVI-LVII.

7 S.A. Immerwahr, Aegean Painting in the Bronze Age, University Park et Londres, 1990, My No. 7, p. 191-192 et pl, 62-63.

$8 \quad$ Ibidem, My No. 21, p. 194 et pl. 84. 
on sait que la tombe A d'Archanes-Phourni, qui offre l'équivalent archéologique du témoignage iconographique du sarcophage, n'appartient pas à la catégorie des tombes circulaires crétoises, dite «tombes de la Mesara", mais bien à celle des tombes à tholos continentales, par sa structure, son élévation et son plan, spécialement avec la chambre latérale, qui n'est attestée que dans le «Trésor d'Atrée», à Mycènes, et dans le «Trésor de Minyas», à Orchomène de Béotie 9 .

Reste le sacrifice du taureau, dont on n'a pas d'exemple dans les tombes mycéniennes. J'ai pu montrer cependant, il y a peu, que l'usage de déposer des rhytons zoomorphes dans les tombes, en particulier des rhytons en forme de tête animale, devait être interprété, selon toute vraisemblance, comme un sacrifice partiel de substitution ${ }^{10}$. Le crâne de taureau d'Archanes-Phourni constitue l'équivalent réel d'une telle pratique. La libation régénératrice de sang qui suit le sacrifice -

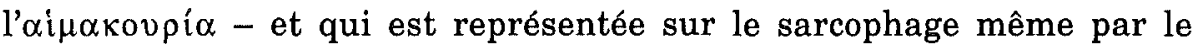
rhyton-cornet enfoncé dans le sol, qui recueille le sang de la victime et lui permet de pénétrer dans la terre, pouvait elle aussi se faire par substitution au moyen du rhyton zoomorphe, qui est avant tout un vase à libation ${ }^{11}$. Il n'est pas inutile de rappeler ici que les contextes de trouvaille des rhytons en forme d'animal ou en forme de tête d'animal sont fondamentalement différents en Crète minoenne et en Grèce mycénienne. Les vases en question n'apparaissent pas avant l'HR I sur le continent grec et ils ont été trouvés en grande majorité dans des tombes; en Crète les vases en tête d'animal se rencontrent dès le MM IIb et proviennent presque exclusivement de contextes cultuels ou $d^{\prime}$ habitat ${ }^{12}$. On verrait volontiers dans cette distinction des contextes de

9 Voir à ce propos O. PELON, Tholoi, tumuli et cercles funéraires, Paris, 1976, p. 365-371.

10 R. LAFFINEUR, Fécondité et pratiques funéraires en Egée à l'âge du Bronze, in Archaeology and Fertility Cult in the Ancient Mediterranean. Papers presented at the First International Conference on Archaeology of the Ancient Mediterranean, Malta, 2-5 September 1985, Amsterdam, 1986, p. 85-88.

11 Voir aussi R. LAFFINEUR, Weitere Beiträge zur Symbolik im mykenischen Bestattungsritual, in Kolloquium zur Ägäischen Vorgeschichte, Mannheim, 20.-22.2. 1986, Schriften des Deutschen Archäologen-Verbandes, IX, Mannheim, 1987, p. 125-132.

12 R.B. KoEHL, The Functions of Aegean Bronze Age Rhyta, in Sanctuaries and Cults in the Aegean Bronze Age. Proceedings of the First International Symposium at the Swedish Institute in Athens, 12-13 May, 1980 Stockholm, 1981, p. 179-188. Voir aussi E.B. MiLler, Zoomorphic Vases in the Bronze Age 
trouvaille une annonce de la dichotomie entre les $\chi 0 \alpha i$, libations en

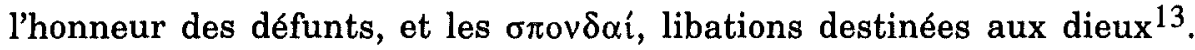
Contrairement à une première impression superficielle, le sarcophage d'Aghia Triada se révèle ainsi illustrer des pratiques qui s'intègrent mieux au domaine mycénien et le monument, que l'on s'accorde à dater de peu après 1400 av. J.-C. ${ }^{14}$, doit donc sans doute être attribué plutôt aux occupants mycéniens de la Crète qu'aux Crétois eux-mêmes, tout comme la tombe A d'Archanes, construite dans la première moitié du XIVe siècle av. J.-C. ${ }^{15} \mathrm{Si}$ même la réalisation du sarcophage a pu être confiée à des artisans minoens ${ }^{16}$, les conceptions et les croyances qui sont à la base de sa décoration peinte ${ }^{17}$ trahissent l'origine de son commanditaire. L'association du rite sacrificiel ou de son substitut aux pratiques funéraires et l'adaptation des formes anciennes du rhyton à une fonction nouvelle dans le cadre de ces mêmes pratiques sont des manifestations claires du syncrétisme créto-mycénien.

Aegean, Ann Arbor, 1984. Les exemplaires minoens trouvés dans des tombes sont extrêmement isolés. On citera le rhyton en tête de taureau de Monasteriako Kephali près de Cnossos, dont la date MR I n'est toutefois pas assurée (MILler [supra n. 12], $\mathrm{n}^{\circ} \mathrm{TC} 99$, p. 397), une pièce analogue de Ligortyno, datée du MR IIIA:2 (E. POTTIER, in BCH, 31 [1907], p. 117, $\mathrm{n}^{\circ} 1$ et pl. XXIII, en haut), et un fragment de la "Tombe des doubles haches» à Cnossos (Sir A. Evans, The Tomb of the Double Axes and Associated Group and Pillar Rooms and Ritual Vessels of the 'Little Palace' at Knossos, Londres, 1914, p. 52 fig. 70).

13 Sur ces deux formes de libations, voir J. RUDHARDT, Notions fondamentales de la pensée religieuse et actes constitutifs du culte dans la Grèce classique, Genève, 1958, p. 240-248.

14 Sur le sarcophage, voir en dernier lieu IMMERWAHR (supra n. 7), p. 100-102.

15 Sur la tombe, voir en dernier lieu I. et E. SAKELlarakis, Archanes, Athènes, 1991, p. 72-85.

16 C'est l'interprétation la plus probable, même si l'usage des sarcophages n'était sans doute pas absent sur le continent helladique aux phases anciennes du Bronze récent, comme on le croit généralement. Voir à ce propos Å. ÅKERström, Mycenaean Problems, in OAth, 12 (1978), p. 60-67.

17 La décoration des petits côtés du sarcophage, avec chaque fois des figures (des divinités ?) en char (MARINATOS-HIRMER, [supra n. 3], pl. XXXII en bas et XXXIII), présente un caractère plus emblématique qui se prête plus difficilement à une interprétation précise. Elle n'a apparemment pas de rapport direct avec les scènes des longs côtés. Sur ce décor, voir en dernier lieu T. SMaLL, $A$ Goat-Chariot on the Hagia Triada Sarcophagus, in AJA, 76 (1972), p. 327 et pl. 72, 1 et J.P. NAUERT, A Goat-Chariot on the Hagia Triada Sarcophagus: a Further Note, in AJA, 76 (1972), p. 437. 
L'endroit de trouvaille et la forme d'un monument n'apparaissent finalement pas aussi déterminants qu'on peut le croire à première vue et c'est essentiel au plan de la méthode. Ils sont en tout cas moins déterminants que l'iconographie, ou plutôt que le contexte particulier dans lequel cette iconographie trouve son interprétation et son sens le plus large et qui peut se révéler différent du contexte de trouvaille et de l'environnement typologique.

La distinction observée plus haut à propos de l'utilisation des vases à libation en Crète et sur le continent mycénien s'applique aussi à la variété particulière des rhytons en forme d'œuf d'autruche. Les exemplaires trouvés en Crète minoenne ou dans les Cyclades proviennent de sites d'habitat ou de sanctuaires ${ }^{18}$, alors que les spécimens mycéniens ont été exhumés dans des tombes, à Mycènes ${ }^{19}$ et à Dendra ${ }^{20}$. Si la signification fondamentale de l'œuf est sans doute la même, à savoir celle d'un symbole de fécondité et de vie, son utilisation particulière est différente dans les deux domaines. Ici encore, les Mycéniens ont emprunté l'usage d'un ustensile, l'œuf d'autruche transformé en vase à libation par l'aménagement d'un orifice de remplissage et le percement d'un trou de vidange, mais ils l'ont adapté à un usage propre dans le cadre du rituel funéraire. La raison de cette libation $a b$ ovo si l'on peut dire et du dépôt de l'œuf dans la tombe après exécution du rituel est évidemment que l'œuf est par excellence, par la vie qu'il contient en germe, une garantie symbolique d'accession à une vie nouvelle dans l'au-delà et qu'il est à ce titre un compagnon idéal du défunt. La taille inhabituelle de l'œuf d'autruche contribue assurément à renforcer l'efficacité de ce symbolisme. Elle n'est qu'une des manifestations de la composante pléonastique qui est typique de la symbolique funéraire mycénienne et qui s'exprime ailleurs par la répétition fréquente du même motif ou par l'association à une même inhumation de plusieurs

18 Voir la liste des trouvailles dans K.P. FOSTER, Aegean Faience of the Bronze Age, New Haven et Londres, 1979, p. 130-134 et J.A. SAKELlaraKis, The Fashioning of Ostrich-Egg Rhyta in the Creto-Mycenaean Aegean, in Thera and the Aegean World III, Proceedings of the Third International Congress, Santorini, Greece, 3-9 September 1989, I, Archaeology, Londres, 1990, p. 286289.

19 Voir les œufs et fragments des tombes IV et $\mathrm{V}$ du cercle de l'acropole : G. KARO, Die Schachtgräber von Mykenai, Munich, 1930-1933, $\mathrm{n}^{\circ} 552,828$, 832.

20 A.W. PERsson, The Royal Tombs at Dendra near Midea, Lund, 1931), p. 37 et 54 et pl. III; ID., New Tombs at Dendra, Lund, 1942, p. 146. 
motifs à valeur analogue dont l'action s'additionne en quelque sorte, au bénéfice de l'efficacité 21 .

Les rhytons mycéniens en forme d'œuf d'autruche s'inscrivent dans un contexte qui n'est pas, pour l'essentiel, différent du contexte des exemplaires minoens. L'origine orientale du matériau est la même ${ }^{22}$; l'addition d'un col et d'un fond en faïence ${ }^{23}$ s'inscrit dans la tradition crétoise, comme celle du motif de spirales enchaînées d'un col en faïence de Mycènes ${ }^{24}$ ou d'une décoration de dauphins en relief en même matière sur un œuf de même provenance ${ }^{25}$, dont on a l'équivalent sur un support en terre cuite MM III de Phaestos ${ }^{26}$. Mais au-delà de ces parentés indéniables, les œufs d'autruche du continent s'inscrivent dans un ensemble cohérent et homogène de conceptions et de pratiques funéraires dont la Crète minoenne n'a pas livré d'attestations, mais qui sont propres au domaine helladique et qui apparaissent dominées par l'idée de régénération et la volonté d'assurer au défunt l'accès à une nouvelle vie dans l'au-delà 27 . On est loin de l'opinion traditionnelle et simpliste qui conclut à l'importation des objets de Crète à Mycènes sur base de la seule attestation de documents analogues en Crète à une

21 Voir à ce propos LAFFineUR (supra n. 10), p. 83-88 et (supra n. 11), p. 125-132.

22 Les œufs d'autruche trouvés en Égée proviennent selon toute vraisemblance de Nubie. Ils font partie en tout cas des offrandes apportées par les Nubiens sur les peintures de la tombe de Rekmiré (voir l'illustration dans Sakellarakis [supra n. 18], p. 305). Le site de Marsa Matru, à l'Ouest d'Alexandrie, qui a livré des œufs d'autruche (D. WHITE, 1985 Excavations on Bates's Island, Marsa Matruh, in JARCE, 23 [1986], p. 79), a probablement servi d'intermédiaire dans le commerce vers l'Égée. Mais l'autruche vivait également en Syrie au IIe millénaire, comme l'indique le témoignage des tablettes de Mari (M. YoN et A. CAUBET, Kition-Bamboula III. Le sondage L-N 13 , Paris, 1985, p. 88). Cette seconde origine semble confirmée par la trouvaille d'œufs d'autruche dans la cargaison du navire qui a fait naufrage au large d'Ulu Burun à la fin du XIVe siècle (G.F. BASs et alii, The Bronze Age Shipwreck at Ulu Burun: 1986 Campaign, in AJA, 93 [1989], p. 9).

23 KARO (supra n. 19), $n^{\circ} 567,573$ et 774.

24 KARO (supra n. 19), $n^{\circ} 567$.

25 KARO (supra n. 19), $\mathrm{n}^{\circ} 828$.

26 Le document est illustré dans SAKELLARAKIS (supra n. 18), fig. 54.

27 Voir à propos de ces préoccupations R. LAFFINEUR, Iconographie minoenne et iconographie mycénienne à l'époque des tombes à fosse, in L'iconographie minoenne. Actes de le Table Ronde d'Athènes (21-22 auril 1983), BCH Suppl. XI (1985), p. 245-266. 
époque plus ancienne. Même si ces objets trouvés sur le continent ont été fabriqués dans des ateliers minoens - la même éventualité n'était pas à exclure plus haut à propos du sarcophage d'Aghia Triada -, il n'en reste pas moins que leur utilisation et leur signification se distinguent nettement de celles des équivalents crétois.

En matière de croyances relatives à l'au-delà, on oppose habituellement la conception d'un au-delà de type élyséen situé aux confins d'une vaste étendue d'eau et auquel on accède au terme d'une longue traversée, que l'on dit naturelle pour un peuple insulaire et qui serait donc propre aux Minoens, et celle plus habituelle de l'au-delà souterrain, annonciateur de l'Hadès, plus typique de populations continentales et donc plus spécifique aux Mycéniens. Qu'une telle dualité, dont on trouve l'expression dans le texte homérique ${ }^{28}$, ait ses racines dans l'Égée préhistorique, comme le suggérait M.P. Nilsson ${ }^{29}$, est une hypothèse certes séduisante et l'origine crétoise de Rhadamanthe, étroitement associé à l'Élysée, de même que l'origine préhellénique de son nom, sont des arguments non négligeables en faveur d'une spécificité minoenne. Mais la réalité archéologique de la Crète minoenne n'apporte pas clairement la confirmation attendue. Tout comme son attestation littéraire ne se fonde que sur un passage isolé du texte homérique, cette interprétation d'un au-delà de type élyséen repose finalement sur des documents uniques, peu nombreux de surcroît. Sans compter les modèles de bateaux en terre cuite, en pierre ou en métal, déposés assez fréquemment dans les tombes ${ }^{30}$ mais dont l'interprétation n'est guère assurée et où il est hasardeux de voir l'image du bateau qui doit "permettre au défunt de traverser l'océan en direction de l'autre monde» 31 , on ne peut invoquer que la scène de la célèbre "bague de Nestor" ou le modèle de barque de la collection Mitsotakis. La première

28 Od. IV, $561 \mathrm{sv}$ : Ménélas conduit à l'Élysée, à l'extrémité de la terre, sur les rivages de l'Océan qui entoure la terre. Sur cette dualité, voir J. WIESNER, Grab und Jenseits. Untersuchungen im ägäischen Raum zur Bronzezeit und frühen Eisenzeit, Berlin, 1938, p. 208-219.

29 M.P. NILsson, The Minoan-Mycenaean Religion and its Survival in Greek Religion, Lund, $1950^{2}$, p. 619-633.

30 L'inventaire en a été dressé par D. GRAX, Seewesen, Archaeologia Homerica

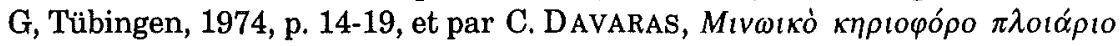

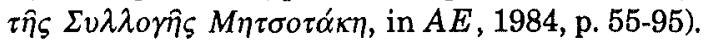

31 Greece and the Sea, catalogue de l'exposition, Amsterdam, 1987, p. 34 (C. Boulotis). 
n'est pas d'une lecture aisée ${ }^{32}$ et même si l'on doit, raisonnablement je crois, abandonner les doutes émis à propos de l'authenticité de la bague $^{33}$, il reste qu'il s'agit d'un témoignage exceptionnel et que l'on souhaiterait disposer au moins d'une seconde illustration comparable avant de conclure que «cet objet paraît bien offrir une vue synthétique de la conception minoenne de l'au-delà, monde centré sur les îles des Bienheureux" ou que "pour accéder à cet Élysée minoen, que domine un lion, il fallait sans doute accomplir, à la manière égyptienne, un grand voyage régénérateur sur la plaine liquide» ${ }^{34}$ ou encore que l'au-delà des Minoens et des gens des Cyclades est transmarin et que "le défunt voyage, comme le montre le sarcophage d'Episkopi, à travers l'océan pour atteindre l'H $H$ v́otov $\pi \varepsilon \delta i ́ o v$, le Champ Élyséen, où réside le Crétois

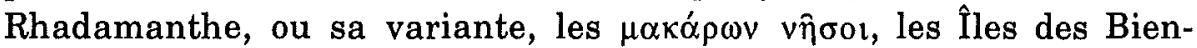
heureux, afin d'y séjourner dans le bien-être» 35 . Quant au modèle de barque de la collection Mitsotakis, il paraît apporter un élément nouveau d'interprétation, puisque le fond de l'embarcation est pourvu, de manière assez inattendue, d'un rayon de miel. Au terme d'un inventaire exhaustif des connotations religieuses du miel, C. Davaras, qui a publié le document de manière approfondie, propose d'interpréter cette partie constituante et le miel qui y était sans doute déposé comme un symbole de la douceur suprême et une allusion au bonheur réservé à l'âme dans l'Élysée ${ }^{36}$. Il ne reste alors que des impressions subjectives,

32 Voir récemment le commentaire du document dans R. HAMPE et E. SIMON, Un millénaire d'art grec, 1600-600, Fribourg, 1980, p. 188-189.

33 Voir J. A. SAKELLARAKIS, Über die Echtheit des sogenannten Nestorringes, in

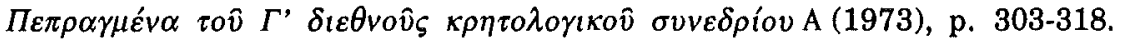
La figure de l'animal à courtes pattes et à longue queue visible au bas de la scène constitue un élément déterminant en faveur de l'authenticité, puisque son équivalent exact se trouve sur une gemme de Mycènes, découverte en 1954 ( $C M S I, n^{\circ} 167$ ) et que l'éventuel faussaire n'aurait donc pas pu connaître.

34 C. BAURAIN, Pour une autre interprétation des génies minoens, in L'iconographie minoenne. Actes de la Table Ronde d'Athènes (21-22 auril 1983), $B C H$, suppl. XI (1985), p. 114.

35 J.VANSCHOONWINKEL, La barque dans le culte et la religion créto-mycéniens, in Revue des archéologues et historiens d'art de Louvain, 15 (1982), p. 30.

36 DAVARAS ( supra n. 30), p. 76-92. Voir aussi Greece and the Sea, catalogue de l'exposition, Amsterdam, 1987, p. 33 : le miel comme nourriture pour le dernier voyage ou comme allusion symbolique au passage de l'âme vers les Champs Élysées, pleins de joie et de douceur après la délivrance des peines d'ici-bas (C. Boulotis). La généralisation de l'interprétation de C. Davaras 
telle la localisation soi-disant préférentielle des sépultures crétoises à proximité de la mer ${ }^{37}$, la forme des sarcophages minoens considérée comme évocatrice de la barque ou du bateau ${ }^{38}$, ou les images de barques ou de bateaux peints sur des sarcophages de la période postpalatiale ${ }^{39}$, dont l'iconographie fait intervenir fréquemment par ailleurs des animaux marins, poulpes, nautiles et poissons ${ }^{40}$.

aux autres modèles de barque est en outre d'autant plus difficile que le document de la collection Mitsotakis présente des particularités inhabituelles, notamment les quatre pieds sur lesquels il est posé, qui n'ont d'équivalent que sur des modèles de la fin du Chypriote récent de Lapithos (L. BASCH, Le musée imaginaire de la marine antique, Athènes, 1987, fig. 313-315, p. 149150), munis d'une anse, et où il convient donc de reconnaître des rhytons.

37 On y verrait volontiers un moyen de rapprocher les défunts du point de départ de leur dernier voyage. Mais une telle localisation est loin de constituer une règle générale. Même si elle est valable et même assez caractéristique pour quelques sites, en particulier pour Malia (C. BAURAIN, Les nécropoles de Malia, in Thanatos. Les coutumes funéraires en Egée à l'âge du Bronze. Actes du colloque de Liège (21-23 avril 1986), Aegaeum 1 [1987], p. 71-72) et, d'une façon plus générale, pour les Cyclades (B. RuTKowsKI, Kykladen und Kreta : Bemerkungen über die bronzezeitliche Religion, in AAA, 9 [1976], p. 237), on montrerait sans peine que les sépultures situées à l'intérieur des terres sont en réalité majoritaires, quels que soient la période ou le domaine du bassin égéen que l'on considère.

Selon B. Rutkowski, la forme même des larnakes serait à considérer comme une allusion symbolique au bateau qui doit permettre au défunt d'effectuer son dernier voyage (RUTKOwSKI [supra n. 37], p. 240-241), mais si l'analogie peut être envisagée lorsqu'il s'agit de sarcophages en forme de cuve ou de baignoire, elle paraît moins adéquate dans le cas des réceptacles funéraires de type coffre. On va même souvent jusqu'à voir dans les motifs de spirales, que l'on trouve sur les "poêles à frire" de Syros et, entre autres, sur le sarcophage d'Aghia Triada, une allusion symbolique complémentaire à la mer qui conduit à l'Élysée (RUTKowsKI [supra n. 37], p. 239-240),. L'interprétation n'est vraiment évidente, on en conviendra, que sur les "poêles à frire", où les spirales sont parfois associées à des images de bateaux, mais il n'est pas assuré que les "poêles à frire» cycladiques aient eu une destination spécifiquement funéraire.

39 Gray (supra n. 30), p. 19. La plus belle illustration se trouve sur le célèbre sarcophage de Gazi (voir en dernier lieu Greece and the Sea, catalogue de l'exposition, Amsterdam, 1987, p. 29).

40 C. MAVRIYANNAKI, Recherches sur les larnakes minoennes de la Crète occidentale, Rome, 1972, passim; K. CzERNOHAUS, Delphindarstellungen von der minoischen bis zur geometrischen Zeit, Göteborg, 1988, p. 59-71 et pl. LVIIILXVI. Pour les poissons, voir en particulier un sarcophage minoen de la nécropole d'Adele (St. HILler, Das minoische Kreta nach den Ausgrabungen 
Quoi qu'il en soit de cette éventualité d'une conception élyséenne de l'au-delà en Crète minoenne, il semble complètement exclu que la même conception ait été reprise par les Mycéniens, qui ne paraissent pas avoir adopté une conception aussi intellectuelle de l'au-delà. Les connotations marines du contexte funéraire mycénien semblent se référer plus simplement à la nature fécondante de l'élément liquide. La mer n'est-elle pas, davantage peut-être que la terre, une source inépuisable de vie ? Même si des croyances analogues n'étaient certes pas absentes en Crète minoenne ${ }^{41}$, une allusion aux propriétés fécondantes de la mer aurait beaucoup mieux sa place dans le contexte funéraire mycénien, au même titre que les allusions symboliques à la renaissance liées à la terre que paraissent indiquer de nombreuses pièces des mobiliers des tombes du début de l'époque mycénienne $e^{42}$. Il n'y aurait là en somme qu'une manifestation complémentaire d'un ensemble cohé-

des letzten Jahrzehnts, Vienne, 1977, fig. 68). Pour le poulpe, voir notamment un sarcophage récemment mis en vente à New York : Egyptian, Near Eastern and Classical Greek and Roman Antiquities, Hesperia Arts Auction, November 27, $1990, \mathrm{II}, \mathrm{n}^{\circ} 45$.

41 C. Boulotis a montré récemment, à la suite d'une analyse iconographique pénétrante, que l'influence de la grande déesse de la nature s'était peut-être étendue aussi au domaine marin (C. Boulotis, La déesse minoenne à la rame-gouvernail, in Proceedings of the 1st International Symposium on Ship Construction in Antiquity (Piraeus, 1985), in Tropis, 1 [1989], p. 59-60). L'association paraît exprimée notamment par la décoration du bandeau d'or de Zakro (L. von MATT, St. Alexiou, N. Platon et H. GuANella, La Crète antique, Paris, 1967, fig. p. 171. À propos de l'authenticité et de la datation du bandeau, voir H.-G. BuCHHOLZ, Ägäische Kunst gefälscht, Acta Praehistorica et Archaeologica 1 [1970], p. 121-122 et R. LAFFINEUR, Le trésor de Zakro :

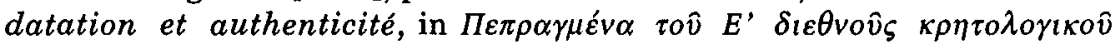

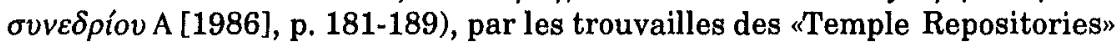
de Cnossos, où les images de la déesse aux serpents étaient accompagnées de coquillages ou de leurs simulacres ainsi que de figures de poisson (K.P. Foster, Aegean Faience of the Bronze Age [1979], p. 78-84, fig. 14 et pl. 13), et, peut-être aussi par le triton en stéatite de Malia (Boulotis, op. cit., p. 59-60). Mais elle est surtout illustrée par la glyptique qui combine le motif du bateau de culte, occupé par la divinité, et celui de l'arbre sacré (sur la bague de Mochlos [CMS II3, $\mathrm{n}^{\circ}$ 252, BouLOTIS, op. cit., fig. 3a], sur la bague dite de Minos [Boulotis, op. cit., fig. $3 \mathrm{~b}$ ] et sur un sceau amygdaloïde de Makrygialos [B ouloTis, op. cit., fig. 3d]) ou des bulbes de scilles de mer, que $P$. Warren a mis en relation avec les cultes de renouveau agraire et avec la déesse de la nature et de la fertilité (Of Squills, in Aux origines de l'hellénisme. La Crète et la Grèce. Hommage à Henri van Effenterre, Paris, 1984, p. 17-24). 
rent de croyances qui associe la composante terrestre et la composante marine en une même volonté d'assurer la survie des défunts ou leur accession à une vie nouvelle dans l'au-delà. Cette préoccupation s'exprimerait tantôt en une simple référence à la mer, par une série de motifs iconographiques à valeur symbolique, poulpe ou triton, que l'on rencontre à Mycènes ${ }^{43}$ et à Peristeria ${ }^{44}$, ou par le dépôt de coquillages ${ }^{45}$, tantôt d'une manière plus active, par l'intermédiaire d'ustensiles spécifiques, les rhytons en forme de triton, qui pouvaient être utilisés, ainsi qu'on l'a suggéré pour le contexte cultuel ${ }^{46}$, pour des libations d'eau de mer exécutées dans le cadre d'un rituel au moment de la mise en terre en vue d'assurer la régénération ${ }^{47}$. Sur ce point précis également, la cohérence et la spécificité des coutumes funéraires mycéniennes se trouve renforcée, en même temps que se confirme la complémentarité des principes fécondants terrestre et marin ${ }^{48}$. Quant aux modèles de barques et aux

43 Le poulpe est représenté au cercle des tombes à fosse de l'acropole : Karo (supra n. 19), $\mathrm{n}^{\circ}$ 18, 30-31, 39-40, 386-387.

44 Voir les images de triton en or de la tholos $3: \mathrm{Sp}$. MARINATos, in SMEA, 3 (1967), p. 12 et fig. 16. Voir aussi les figures d'argonaute sur la parure funéraire en or ou en pâte de verre : R. LAFFINEUR, Mycenaean Artistic Koine : the Example of Jewelry, in Temple University Aegeazn Symposium, 9 (1984), p. $9-11$, n. 14 (et la carte de distribution, p. 3 (il faut ajouter aujourd'hui d'autres exemplaires des tombes à chambre de Mycènes publiés dans A. Xenaki-SAKellariou, Les tombes à chambre de Mycènes. Fouilles de Chr. Tsountas 1887-1898, Paris, 1985, p. 308, type 118 et pl. 108, $\mathrm{n}^{\circ} 3127 / 10$ [tombe 81 ] et $136, n^{\circ} 5413 / 2$ [tombe 100]).

45 Pour cette catégorie de trouvailles, attestées également en Crète et dans les Cyclades, voir D.S. REESE, in ABSA, 77 (1982), p. 249-250 (Gypsades, tombe XVIII; référence à des trouvailles analogues dans une tombe $\mathrm{CA}$ de Lakkoudhes à Naxos et une tombe HM de Prosymna); ABSA, 78 (1983), p. 353-357 (tombes à chambre de Mycènes, Tirynthe, Prosymna et Perati). Pour les Cyclades, voir C. Doumas, Early Bronze Age Burial Habits in the Cyclades, Göteborg, 1977, passim.

46 BAURAIN (supra n. 34), p. 115; Boulotis (supra n. 41), p. 60 (et n. 29 à propos de l'utilisation comme vase à libation).

47 Le rhyton en forme de poisson avait peut-être la même destination. Voir un spécimen HR III B de Tirynthe (Greece and the Sea, catalogue de l'exposition, Amsterdam, 1987, $n^{\circ} 51$, p. 152) et des exemplaires de Minet-el-Beida au Louvre (MILler [supra n. 12], p. 433-434 et pl. 298-300).

48 Cette dualité et cette complémentarité ne sont-elles pas exprimées de manière particulièrement suggestive sur un sarcophage de Pachyammos où l'image du poulpe voisine avec celle du papyrus (MARINATOS-HIRMER [supra n. 3], pl. 130 , en haut)? 
figurations de bateaux peintes sur les sarcophages, ils ne constitueraient pas davantage qu'une allusion abrégée à la mer au même titre que le décor d'ondulations couvrant la cuve ${ }^{49}$ ou que les images empruntées à la faune marine ${ }^{50}$. Cela n'empêche pas que la référence puisse être spécialement suggestive. Ainsi, la position des motifs sur les sarcophages n'est peut-être pas toujours le fait du hasard, comme tend à le laisser supposer le fait que les figures de dauphins et de poissons ornent la plupart du temps la face intérieure de la paroi verticale du réceptacle funéraire ${ }^{51}$ et les ondulations quelquefois la face intérieure $\mathrm{du}$ fond ${ }^{52}$, comme si on avait voulu les rapprocher davantage encore du défunt, ou même en quelque sorte identifier à la mer le volume du sarcophage dans lequel il est déposé.

L'iconographie des sarcophages de la période postpalatiale montre ainsi une prédilection pour les motifs marins qui paraît n'être pas seulement liée à la composante maritime générale du monde minoen, mais plus précisément à des conceptions et des rites funéraires qui privilégient la valeur fécondante de la mer et qui paraissent ainsi étroitement associés à ceux qui sont fondés sur la fécondité de la terre et

Sur un sarcophage MR III A de la tombe A d'Archanes-Phourni (HILleR [supra n. 40], fig. 61) et des sarcophages de Pachyammos et de Milatos (A. KANTA, The Late Minoan III Period in Crete. A Survey of Sites, Pottery and their Distribution, Göteborg, 1980, pl. 52, 4-5).

50 Cette interprétation paraît en tout cas préférable à celle d’O. Höckmann qui, observant que les quatre modèles de bateau en plomb d'une tombe de Naxos se répartissent sans doute par moitiés entre les deux occupants de la sépulture et sont donc plus nombreuses que la seule embarcation qui serait nécessaire à chacun d'eux pour assurer leur voyage dans l'au-delà, conclut qu'il s'agit plutôt de «Grabschiffe mit denen ein reicher Schiffseigner oder Fürst auch im Jenseits eine standesgemäße Lebensweise fortsetzen sollte" (Kunst und Kultur der Kykladeninseln im 3. Jahrtausend v. Chr., catalogue de l'exposition, Karlsruhe, 1976, p. 48).

51 Czernohaus [supra n. 40], pl. LVIII, 3; LIX, 4; LX, 1; LXI, 1; LXV, 1, 3 et 5; LXVI, 2; KANTA (supra n. 49), pl. 56, 3; 65, 4; 66, 2; 73, 10. L'observation a déjà été faite en partie dans I. PINI, Beiträge zur minoischen Gräberkunde, Wiesbaden, 1968, p. 54 et dans J. ThImme, La civilsation des Cyclades à l'âge du Bronze ancien, in J. Thimme, P. Åström, G. Lilliu et J. Wiesner, Civilisations anciennes du bassin méditerranéen. Les Cyclades, Chypre, Malte, la Syrie ancienne, Paris, 1971, p. 55.

52 Czernohaus [supra n. 40], pl. LXV, 3 et 5; LXVI, 2. L'intérieur des sarcophages est rarement reproduit et il est possible que cette décoration soit en réalité plus fréquente. 
qui semblent bien constituer une composante spécifiquement mycénienne. L'association de motifs végétaux et de motifs marins est ici significative. Le syncrétisme joue ici en sens inverse. Je vous invitais en commençant à un voyage de Cnossos à Mycènes. Le parcours était en réalité plus long, puisqu'il s'agit en définitive d'un voyage «de Cnossos à Mycènes et retour".

Robert LAFFINEUR

Université de Liège

Archéologie grecque

Place du 20-Aout, 32

B - 4000 LIÈGE 